Proceedings of the MACPROGEN Final Conference held at Ohrid, Republic of Macedonia, March 29-April 12012

\title{
INTEGRATED GENOMIC ANALYSIS OF BREAST CANCERS
}

Addou-Klouche $\mathrm{L}^{1,2}$, Adélaïde ${ }^{1} \mathrm{~J}$, Cornen $\mathrm{S}^{1}$, Bekhouche $\mathrm{I}^{1}$, Finetti $\mathrm{P}^{1}$, Guille $\mathrm{A}^{1}$, Sircoulomb F ${ }^{1}$, Raynaud $\mathrm{S}^{1}$, Bertucci $\mathrm{F}^{1,3,4}$, Birnbaum $\mathrm{D}^{1}$, Chaffanet $\mathrm{M}^{1, *}$

*Corresponding Author: Max Chaffanet, Ph.D., HDR, Department of Molecular Oncology, Institut Paoli Calmettes, 232 Boulevard Sainte Marguerite, 13009 Marseille, France; Tel.: +33-(0)4-91-22-34-77; Fax: +33(0)4-91-22-35-44; E-mail: CHAFFANETM@ipc.unicancer.fr

\begin{abstract}
Breast cancer is the most frequent and the most deadly cancer in women in Western countries. Different classifications of disease (anatomoclinical, pathological, prognostic, genetic) are used for guiding the management of patients. Unfortunately, they fail to reflect the whole clinical heterogeneity of the disease. Consequently, molecularly distinct diseases are grouped in similar clinical classes, likely explaining the different clinical outcome between patients in a given class, and the fact that selection of the most appropriate diagnostic or therapeutic strategy for each patient is not done accurately. Today, treatment is efficient in only 70.0$75.0 \%$ of cases overall. Our repertoire of efficient drugs is limited but is being expanded with the discovery of new molecular targets for new drugs, based on the identification of candidate oncogenes and tumor suppressor genes (TSG) functionally relevant in disease. Development of new drugs makes
\end{abstract}

1 Marseille Cancer Research Center, Department of Molecular Oncology, UMR891 Inserm; Institut Paoli-Calmettes, Marseille, France

2 Biotoxicology Laboratory, Djillali Liabes University, Sidi-Bel-Abbès, Algeria

3 Department of Medical Oncology, Institut PaoliCalmettes, Marseille, France

$4 \quad$ Université de la Méditerranée, Marseille, France therapeutical decisions even more demanding of reliable classifiers and prognostic/predictive tests. Breast cancer is a complex, heterogeneous disease at the molecular level. The combinatorial molecular origin and the heterogeneity of malignant cells, and the variability of the host background, create distinct subgroups of tumors endowed with different phenotypic features such as response to therapy and clinical outcome. Cellular and molecular analyses can identify new classes biologically and clinically relevant, as well as provide new clinically relevant markers and targets.

The various stages of mammary tumorigenesis are not clearly defined and the genetic and epigenetic events critical to the development and aggressiveness of breast cancer are not precisely known. Because the phenotype of tumors is dependent on many genes, a large-scale and integrated molecular characterization of the genetic and epigenetic alterations and gene expression deregulation should allow the identification of new molecular classes clinically relevant, as well as among the altered genes and/or pathways, the identification of more accurate molecular diagnostic, prognostic/predictive factors, and for some of them, after functional validation, the identification of new therapeutic targets.

Keywords: Breast cancers, Genome, Transcriptome, Epigenome, Oncogenes, Tumor suppressor genes 


\section{INTRODUCTION}

High-Throughput Molecular Analyses in Breast Cancer and Translational Research. Unprecedented molecular characterization is possible using high-throughput molecular analyses, available at the DNA level with comparative genomic hybridization on microarrays (aCGH) [1-4], and at the RNA level, for expression profiling with DNA microarrays [5]. When these techniques emerged, expected applications were multiple in oncology, in both basic and translational research.

A number of studies have already shown the promising role of DNA microarray-based expression profiling in breast cancer translational research by identifying new clinically and biologically relevant intrinsic molecular subtypes (luminal A, luminal B, ERBB2+, basal, and normal-like) [6-7] and new prognostic and/or predictive gene signatures, whose predictive impact is superior to conventional histoclinical factors (for review, see [8]). Currently, three prognostic gene signatures are already commercially available: Oncotype DX (Genomic Health, Inc., Redwood City, CA, USA), MammaPrint (Agendia BV, Amsterdam, The Netherlands), and the HOXB13/ IL17BR (H/I) ratio (Theros H/ISM; bioTheranostics, San Diego, CA, USA). Others under development include the Intrinsic Gene Set, the Rotterdam Signature, the Wound Response Indicator, and the Invasive Gene Signature. Similarly, signatures predictive for response to specific therapies have been reported [9-12]. These prognostic or predictive signatures, once prospectively validated, will provide the opportunity to refine our therapeutic approach by individualizing treatment to patients'individual tumor profiles, likely contributing to significantly improve the clinical outcome (for review, see [13]).

The aCGH technology has been applied more recently to breast cancer. To date, some studies, including ours, have suggested a prognostic role of genomic data [14-16]. The integrative analysis of whole-genome expression and genomic data has revealed promising results for identifying candidate genes (identified as deregulated at the DNA and RNA levels simultaneously) associated with breast cancer or with specific features of disease [14,16-24].

For years, our laboratory has identified a large number of molecular alterations in recurrent breast cancer associated with: $\boldsymbol{i}$ ) structural aberrations such as breakages [25-29], and ii) evaluated the clinical impact of the amplification [14,30,31]. We were among the first to demonstrate that the integrative analysis of whole-genome expression and genomic high resolution data are useful to identify new oncogenes and TSG specific to a clinical entity or a molecular subtype. Therefore, our comparative analyses of integrated profiles of breast cancers have been reported in basal and luminal tumors, two molecular subtypes of very different clinical courses [19], but also in particularly aggressive cancer: inflammatory breast cancer [32], breast cancers in young women (Raynaud et al., in preparation), and ERBB2 amplified breast cancers [33]. This laboratory was also one of the first to identify specific genomic markers of luminal B: L3MBTL4 (18p11) [34] and ZNF703 (8p12) [35] as potential TSG and oncogene, respectively.

Candidate Genes May Also be Transcriptionnally Deregulated Because of Epigenetic Alterations. The widespread deregulation of basic epigenetic profiles has emerged as a common phenotypic trait of cancer cells [36-38]. The epigenetic modifications include covalent tags added to nucleosome histone components [e.g., acetylation of histone $\mathrm{H} 3$ and/or H4 (H3/4Ac) and/or various levels of methylation on lysine residues of histone $\mathrm{H} 3$ (H3K4/ $\mathrm{K} 9 \mathrm{me} 1 / 2 / 3$ ), a non exhaustive list defined as the histone code], as well as methylation of $\mathrm{CpG}$ dinucleotides $[39,40]$. This applies particularly to $\mathrm{CpG}$ methylation profiles, whose modification has direct implication on many aspects of cell biology, namely cell division, survival, development and, consequently, oncogenesis. DNA methylation at regulatory regions of a gene, including promoter, generally leads to transcriptional silencing. $\mathrm{CpG}$ methylation-dependent silencing is now considered as an important mechanism of TSG inactivation in cancer cells, in addition to somatic genetic lesions [41]. DNA methylation changes in human cancers are complex and vary between different tumor types. Promoter methylation effectively represses transcription and occurs in many genes involved in human breast cancer development [42]. Among these, genes associated with cell cycle regulation (APC, RASSF1, RB, TFAP2A), or coding for steroid receptors (ESR1, PGR, RAR $\alpha$ ), suppressors (BRCA1, CDKN2A, CST6), and genes associated 
with metastasis (CDH1, CEACAM6, PCDHGB6) and other genes such as NRG1. The majority of these affected genes are potential or known TSG [43]. Interestingly, there is also increasing evidence that methylation of regulatory regions of cancer-related genes can be one of the most prevalent molecular markers for human cancer diseases [44]. The potential clinical applications of DNAmethylation biomarkers may include diagnosis of neoplasm, tumor classification, prediction of response to treatment, or prognosis. DNA methylation status has thus been extensively studied in various molecular or clinical entities in breast cancers in order to better characterize them or improve their molecular classification [45-49].

In the continuity of our strategy, the high resolution DNA promoter methylation status will be analyzed on human promoter array (Agilent Technologies, Massy, France) and integrated to the genomic and gene expression data previously collected in the same set of 300 breast tumors. Highthroughput molecular analyses of breast cancer have already revealed some part of their potential. Such integrated approaches could contribute to better understand the various levels of the dynamic molecular changes in the mammary oncogenesis and identify new markers.

\section{REFERENCES}

1. Solinas-Toldo S, Lampel S, Stilgenbauer S, et al. Matrix-based comparative genomic hybridization: biochips to screen for genomic imbalances.Genes Chromosomes Cancer. 1997; 20(4): 399-407.

2. Pinkel D, Segraves R, Sudar D, et al. High resolution analysis of DNA copy number variation using comparative genomic hybridization to microarrays. Nat Genet. 1998; 20(2): 207-211.

3. van Beers EH, Nederlof PM. Array-CGH and breast cancer. Breast Cancer Res. 2006; 8(3): 210-219.

4. Curtis C, Lynch AG, Dunning MJ, et al. The pitfalls of platform comparison: DNA copy number array technologies assessed. BMC Genomics. 2009; 10: 588-610.

5. Bertucci F, Houlgatte R, Nguyen C, Viens P, Jordan BR, Birnbaum D. Gene expression profiling of cancer by use of DNA arrays: how far from the clinic? Lancet Oncol. 2001; 2(11): 674-682.

6. Perou CM, Sørlie T, Eisen MB, et al. Molecular portraits of human breast tumours. Nature. 2000; 406 (6797): 747-752.
7. Sørlie T, Perou CM, Tibshirani R, et al. Gene expression patterns of breast carcinomas distinguish tumor subclasses with clinical implications. Proc Natl Acad Sci USA. 2001; 98(19): 10869-10874.

8. Sotiriou C, Pusztai L. Gene-expression signatures in breast cancer. N Engl J Med. 2009; 360(8): 790-800.

9. Chang JC, Wooten EC, Tsimelzon A, et al. Gene expression profiling for the prediction of therapeutic response to docetaxel in patients with breast cancer. Lancet. 2003; 362(9381):362-369.

10. Ayers M, Symmans WF, Stec J, et al. Gene expression profiles predict complete pathologic response to neoadjuvant paclitaxel and fluorouracil, doxorubicin, and cyclophosphamide chemotherapy in breast cancer. J Clin Oncol. 2004; 22(12): 2284-2293.

11. Bonnefoi H, Potti A, Delorenzi M, et al. Validation of gene signatures that predict the response of breast cancer to neoadjuvant chemotherapy: a substudy of the EORTC 10994/BIG 00-01 clinical trial. Lancet Oncol. 2007; 8(12): 1071-1078.

12. Farmer $\mathrm{P}$, Bonnefoi $\mathrm{H}$, Anderle $\mathrm{P}$, et al. A stromarelated gene signature predicts resistance to neoadjuvant chemotherapy in breast cancer. Nat Med. 2009; 5(1): 68-74.

13. Dunn L, Demichele A. Genomic predictors of outcome and treatment response in breast cancer. Diagn Mol Ther. 2009; 13(2): 73-90.

14. Gelsi-Boyer V, Trouplin V, Adélaïde J, et al. Genome profiling of chronic myelomonocytic leukemia: frequent alterations of RAS and RUNX1 genes. BMC Cancer. 2008; 8: 299-313.

15. Bergamaschi A, Kim YH, Wang $\mathrm{P}$, et al. Distinct patterns of DNA copy number alteration are associated with different clinicopathological features and gene-expression subtypes of breast cancer. Genes Chromosomes Cancer. 2006; 45(11): 1033-1040.

16. Chin K, de Vries S, Fridlyand J, et al. Genomic and transcriptional aberrations linked to breast cancer pathophysiologies. Cancer Cell. 2006; 10(6): 529541.

17. Stransky N, Vallot C, Reyal F, et al. Regional copy number-independent deregulation of transcription in cancer. Nat Genet. 2006; 38(12): 1386-1396.

18. Neve RM, Chin K, Fridlyand J, et al. A collection of breast cancer cell lines for the study of functionally distinct cancer subtypes. Cancer Cell. 2006; 10(6): 515-527.

19. Adélaïde J, Finetti P, Bekhouche I, et al. Integrated profiling of basal and luminal breast cancers. Cancer Res. 2007; 67(24): 11565-11575.

20. Chin SF, Teschendorff AE, Marioni JC, et al. Highresolution aCGH and expression profiling identifies a novel genomic subtype of ER negative breast cancer. Genome Biol. 2007; 8(10): R215-R231. 
21. Bernard-Pierrot I, Gruel N, Stransky N, et al. Characterization of the recurrent 8p11-12 amplicon identifies PPAPDC1B, a phosphatase protein, as a new therapeutic target in breast cancer. Cancer Res. 2008; 68(17): 7165-7175.

22. Vincent-Salomon A, Lucchesi C, Gruel N, et al. Integrated genomic and transcriptomic analysis of ductal carcinoma in situ of the breast. Clin Cancer Res. 2008; 14(7): 1956-1965.

23. André F, Job B, Dessen P, et al. Molecular characterization of breast cancer with high-resolution oligonucleotide comparative genomic hybridization array. Clin Cancer Res. 2009; 15(2): 441-451.

24. Horlings HM, Lai C, Nuyten DS, et al. Integration of DNA copy number alterations and prognostic gene expression signatures in breast cancer patients. Clin Cancer Res. 2010; 16(2): 651-663.

25. Popovici C, Basset C, Bertucci F, et al. Reciprocal translocations in breast tumor cell lines: cloning of a $\mathrm{t}(3 ; 20)$ that targets the FHIT gene. Genes Chromosomes Cancer. 2002; 35(3): 204-218.

26. Adélaïde J, Huang HE, Murati A, et al. A recurrent chromosome translocation breakpoint in breast and pancreatic cancer targets the heregulin/NRG1 gene at 8p12. Genes Chromosomes Cancer. 2003; 37(4): 333-345.

27. Huang HE, Chin S-F, Ginestier C, et al. A recurrent chromosome breakpoint in breast cancer at the NRG1/ neuregulin 1/heregulin gene. Cancer Res. 2004; 64(19): 6840-6844.

28. Letessier A, Ginestier C, Charafe-Jauffret E, et al. ETV6 gene rearrangements in invasive breast carcinoma. Genes Chromosomes Cancer. 2005; 44(1): 103-108.

29. Letessier A, Garrido-Urbani S, Ginestier C, et al. Correlated break at PARK2/FRA6E and loss of AF6/ Afadin protein expression are associated with poor outcome in breast cancer. Oncogene. 2007; 26(2): 298-307.

30. Ginestier C, Bardou V-J, Popovici C, et al. Absence of FHIT protein expression correlates with adverse evolution in good prognosis localized breast cancer. Int J Cancer. 2003; 107(5): 854-862.

31. Letessier A, Sircoulomb F, Ginestier C, et al. Frequency, prognostic impact, and subtype association of 8p12, 8q24, 11q13, 12p13, 17q12, and 20q13 amplifications in breast cancers. BMC Cancer. 2006; 6(1): 245-257.

32. Bekhouche I, Finetti P, Adélaïde J, et al. Genome profiling of inflammatory breast cancer. PLoS One. 2011; 6(2): e16950-16962.

33. Sircoulomb F, Bekhouche I, Finetti P, et al. Genome profiling of ERBB2-amplified breast cancers. BMC Cancer. 2010; 10: 539-556.
34. Addou-Klouche L, Adélaïde J, Finetti P, et al. Loss, mutation and deregulation of L3MBTL4 in breast cancers. Mol Cancer. 2010; 9(1): 213-225.

35. Sircoulomb F, Nicolas N, Ferrari A, et al. ZNF703 gene amplification at 8p12 specifies luminal B breast cancer. EMBO Mol Med. 2011; 3(3): 153-166.

36. Jones PA, Baylin SB. The fundamental role of epigenetic events in cancer. Nat Rev Genet. 2002; 3(6): 415-428.

37. Lund AH, van Lohuizen M. Epigenetics and cancer. Genes Dev. 2004; 18(19): 2315-2335.

38. Rice KL, Hormaeche I, Licht JD. Epigenetic regulation of normal and malignant hematopoiesis. Oncogene. 2007; 26(47): 6697-6714.

39. Goll MG, Bestor TH. Histone modification and replacement in chromatin activation. Genes Dev. 2002; 16(14): 1739-1742.

40. Klose RJ, Bird AP. Genomic DNA methylation: the mark and its mediators. Trends Biochem Sci. 2006; 31(2): 89-97.

41. Esteller M. Epigenetic gene silencing in cancer: the DNA hypermethylome. Hum Mol Genet. 2007; 16(Spec 1): R50-R59.

42. Wildschwendter M, Jones PA. DNA methylation and breast carcinogenesis. Oncogene. 2002; 21(35): 5462-5482.

43. Mulero-Navarro S, Esteller M. Epigenetic biomarkers for human cancer: The time is now. Crit Rev Oncol Hematol. 2008; 68(1): 1-11.

44. Ordway JM, Budiman MA, Korshunova Y, et al. Identification of novel high-frequency DNA methylation changes in breast cancer. PLoS One. 2007; 2(12): e1314-1325.

45. Bediaga NG, Acha-Sagredo A, Guerra I, et al. DNA methylation epigenotypes in breast cancer molecular subtypes. Breast Cancer Res. 2010; 12(5): R77-R78.

46. Holm K, Hegardt C, Staaf J, et al. Molecular subtypes of breast cancer are associated with characteristic DNA methylation patterns. Breast Cancer Res. 2010; 12 (3): R36-R51.

47. van der Auwera I, Yu W, Suo L, et al. Array-based DNA methylation profiling for breast cancer subtype discrimination. PLoS One. 2010; 5(9): e1261612625.

48. Fackler MJ, Umbricht CB, Williams D, et al. Genome-wide methylation analysis identifies genes specific to breast cancer hormone receptor status and risk of recurrence. Cancer Res. 2011; 71(19): 6195 6207.

49. Hill VK, Ricketts C, Bieche I, et al. Genome-wide DNA methylation profiling of $\mathrm{CpG}$ islands in breast cancer identifies novel genes associated with tumorigenicity. Cancer Res. 2011; 71(8): 2988-2999. 